EPJ Web of Conferences 97,00014 (2015)

DOI: 10.1051/epjconf/20159700014

(C) Owned by the authors, published by EDP Sciences, 2015

\title{
Vector Meson Spectral Functions in a Coarse-Graining Approach
}

\author{
Stephan Endres ${ }^{1,2 a}$, Hendrik van Hees ${ }^{1,2}$, Janus Weil ${ }^{1,2}$, and Marcus Bleicher ${ }^{1,2}$ \\ ${ }^{1}$ Institut für Theor. Physik, Universität Frankfurt, Max-von-Laue-Straße 1, D-60438 Frankfurt, Germany \\ ${ }^{2}$ Frankfurt Institute for Advanced Studies, Ruth-Moufang-Straße 1, D-60438 Frankfurt, Germany
}

\begin{abstract}
Dilepton production in heavy-ion collisions at top SPS energy is investigated within a coarse-graining approach that combines an underlying microscopic evolution of the nuclear reaction with the application of medium-modified spectral functions. Extracting local energy and baryon density for a grid of small space-time cells and going to each cell's rest frame enables to determine local temperature and chemical potential by application of an equation of state. This allows for the calculation of thermal dilepton emission. We apply and compare two different spectral functions for the $\rho$ : A hadronic many-body calculation and an approach that uses empirical scattering amplitudes. Quantitatively good agreement of the model calculations with the data from the NA60 collaboration is achieved for both spectral functions, but in detail the hadronic many-body approach leads to a better description, especially of the broadening around the pole mass of the $\rho$ and for the low-mass excess. We further show that the presence of a pion chemical potential significantly influences the dilepton yield.
\end{abstract}

\section{Introduction}

Although lepton pairs came into the focus of interest as probes for the creation of a deconfined phase [1-3], the so-called quark-gluon plasma (QGP), the hadronic contributions to dilepton production have become a field of vigorous theoretical investigation on their own [4-7]. As vector mesons directly couple to the electromagnetic current and can convert into a lepton pair, the change of their spectral shape inside hot and dense matter can be studied by measuring invariant-mass spectra of dileptons. These properties of hadrons in the medium are of immanent interest to achieve a full understanding of the phase structure given by quantum chromodynamics (QCD). Detailed investigations might help to understand how the high-energy regime with partonic degrees of freedom is connected to the non-perturbative low-energy part, where quarks and gluons are confined in hadrons. Another aspect of study is the symmetry pattern of QCD which is expected to change from its vacuum properties when going to finite temperature and baryochemical potential. In particular, the restoration of chiral symmetry (which is spontaneously broken in the vacuum) is predicted. However, a theoretical and experimental clarification of these issues is associated with several theoretical challenges [6-8]:

- Due to the fact that leptons do not interact strongly, dilepton spectra always represent an integral over the whole evolution of the collision. In the course of the reaction, dileptons can be produced in many different ways. Therefore one has to identify the relevant sources and disentangle a large number of contributions, which is by no means trivial.

\footnotetext{
a e-mail: endres@ fias.uni-frankfurt.de
} 
- Consequently, the dilepton production is also highly influenced by the reaction dynamics. This concerns the lifetime and expansion of the created fireball but also the spatial distribution of temperature and density. Furthermore, the question if and how fast the system will equilibrate is of importance.

- As the reactions in a heavy-ion collision are governed by the strong interaction, one has to deal with complications such as multi-particle collisions, finite temperature and density corrections to form factors and widths, off-shell dynamics, etc.

These aspects impose high requirements on theoretical descriptions that model the dilepton production in heavy-ion collisions. It is necessary (i) to identify the relevant contributions to the dilepton yield, (ii) find a realistic description of the space-time evolution of the reaction and (iii) implement the medium modifications in an appropriate way.

While the dilepton sources at SPS energies are more or less uncontentious, there exist several different approaches providing a theoretical description of the reaction dynamics. In fireball models $[9,10]$ the zone of hot and dense matter is described by an isentropically expanding cylindrical volume. Here an implementation of medium effects is straightforward via the application of spectral functions from thermal quantum field theory. But such a fireball parametrization gives a simplified picture of the reaction, with one value for temperature $T$ and baryochemical potential $\mu_{B}$ in the whole volume. A more nuanced description is delivered by hydrodynamic models $[11,12]$ respectively a hydro+transport hybrid model [13]. The difficulty here is that one usually needs some initial state for the start of the hydrodynamic evolution and additionally some description of the final-state interactions. Furthermore, it is questionable whether such approaches will also work at low energies, e.g. for SIS 18 energies.

A completely different ansatz is used in transport models [14-20]. These approaches treat the dynamics microscopically and account for non-equilibrium, but the implementation of full medium effects, which were discussed above (i.e. multi-particle collisions, off-shell effects, etc.), is difficult here. Nevertheless, there have been some successful investigations with medium-effects implemented in transport models [16, 21-23]. However, problems such as the large variety of parameters, especially cross-sections and branching ratios for which little data is available, remain.

The idea of the present work is to combine a realistic microscopic description of the reaction with an application of medium-modified spectral functions ${ }^{1}$. Ideally, it is applicable at all stages of the reaction so that one has a unified description well-working for different collision energies (i.e. from SIS to RHIC or LHC). This led to the development of the coarse-graining approach as presented in these proceedings, following an ansatz proposed in [24].

This paper is structured as follows: Section 2 introduces the coarse-graining approach while section 3 gives an overview of the spectral functions, discussing the various dilepton sources that enter the calculation. An overview of the results for top SPS energy and a comparison with the experimental dilepton spectra from the NA60 Collaboration is presented in section 4. Finally, a summary and outlook is given in section 5 .

\section{The Coarse-Graining Approach}

To combine a realistic microscopic (3+1)-dimensional description of the colliding system with full in-medium spectral functions, one first has to extract the local thermodynamic properties from the underlying simulations. For the present study, we use input from the Ultra-relativistic Quantum Molec-

\footnotetext{
${ }^{1}$ As we extract only baryon, pion and energy density for each space-time cell, we are not that sensitive with regard to all the details of the model parameters (as for example branching ratios) as an explicit transport-only calculation would be. Furthermore, we avoid the difficulties which a full off-shell description of the reaction would imply.
} 
ular Dynamics (UrQMD) approach [25-27]. It is a non-equilibrium model which includes baryonic and mesonic degrees of freedom with masses up to $2 \mathrm{GeV} / c^{2}$. The hadrons are propagated on classical trajectories, and the particle production is described by inelastic cross sections. String excitation is possible for collisions with $\sqrt{s}>3 \mathrm{GeV}$.

To obtain the thermodynamic properties from UrQMD calculations, it is necessary to average over a sufficiently large number of events. For the present study we use an ensemble of 1000 UrQMD events. Then one finds a (relatively) smooth form of the particle distribution function,

$$
f(\vec{x}, \vec{p}, t)=\left\langle\sum_{h} \delta^{3}\left(\vec{x}-\vec{x}_{h}(t)\right) \delta^{3}\left(\vec{p}-\vec{p}_{h}(t)\right)\right\rangle .
$$

by summing over the phase-space contributions of each hadron $h$ and taking the ensemble average. As the underlying dynamics is due to a non-equilibrium model, it is only locally and only approximately possible to extract the equilibrium properties. In practice we obtain local energy and baryon density by setting up a space-time grid of small cells ${ }^{2}$ with dimensions $\Delta x=\Delta y=\Delta z=0.8 \mathrm{fm}$ and a timestep with $\Delta t=0.2 \mathrm{fm} / \mathrm{c}$. For each cell, the averaged baryon four-flow $j_{\mathrm{B}}^{\mu}$ and the energy-momentum tensor $T^{\mu \nu}$ can be determined as

$$
\begin{aligned}
T^{\mu \nu} & =\int \mathrm{d}^{3} p \frac{p_{\mu} p_{v}}{p_{0}} f(\vec{x}, \vec{p}, t)=\frac{1}{\Delta V}\left\langle\sum_{i=1}^{N_{h} \in \Delta V} \frac{p_{\mu}^{i} \cdot p_{v}^{i}}{p_{0}^{i}}\right\rangle, \\
j_{\mu}^{\mathrm{B}} & =\int \mathrm{d}^{3} p \frac{p_{\mu}}{p_{0}} f^{\mathrm{B}}(\vec{x}, \vec{p}, t)=\frac{1}{\Delta V}\left\langle\sum_{i=1}^{N_{\mathrm{B} / \mathrm{B}} \in \Delta V} \pm \frac{p_{\mu}^{i}}{p_{0}^{i}}\right\rangle .
\end{aligned}
$$

According to Eckart's definition, we can then find the local rest frame of the cell by performing a Lorentz boost to a frame, where $\vec{j}_{\mathrm{B}}=0$. The energy-momentum tensor should then be diagonal and we can identify the energy density $\varepsilon$ with $T^{00}$ while the baryon density is given by $j_{\mathrm{B}}^{0}$. The assumption of an isotropic equilibrated thermal system can be checked by looking at the diagonal matrix elements $T^{i i}(i \in\{1,2,3\})$ of the energy-momentum tensor which represent the transverse and longitudinal pressures. While after a certain time they are usually found to be roughly equal, at the very beginning of the collision (for top SPS energy the first $1-2 \mathrm{fm} / c^{2}$ ) one observes large pressure differences. It is clear that when the two nuclei start to interact and traverse each other at first a strong longitudinal pressure builds up before the system becomes more or less isotropic at later times. To account for this, we apply a description developed for anisotropic hydrodynamics [28] which translates the anisotropic momentum distribution into an equilibrium picture and gives a realistic value of the "effective" energy density in the cell. Applying this procedure allows to use the coarse-graining approach over the whole evolution of the collision.

Once energy and baryon density in the cell are known, we need an equation of state (EoS) to determine temperature and baryochemical potential. For consistency with the underlying microscopic dynamics we employ a hadron-gas EoS [29] which includes the same degrees of freedom as the UrQMD model. However, as we consider a collision energy where the expected temperatures will be high enough to create a deconfined phase of quarks and gluons, a pure hadron gas will not give the

\footnotetext{
${ }^{2}$ Note that with the size of the cells and the number of events chosen for the calculation, we get an approximate error in baryon density of roughly $10 \%$ for $\rho=0.5 \rho_{0}$, and of the same order also for energy density $\varepsilon$. This guarantees a sufficient accuracy of the simulations. In cells with higher densities, which are the dominant sources of thermal dileptons, one will find even less fluctuations. The error especially in temperature will be quite small, as $T \propto \varepsilon^{1 / 4}$. Furthermore, any fluctuations will be averaged out in the final (space-time integrated) dilepton spectra so that the overall statistical effect on the thermal yields is rather negligible. To obtain sufficient statistics for the non-thermal $\rho$ contribution (see below) several coarse-graining runs with different ensembles of UrQMD events as input had to be performed.
} 
correct description for high energy densities. Therefore we use a second EoS obtained from a lattice fit [30] to take the (cross-over) phase transition into account. The critical temperature $T_{c}$ is here found to be at $170 \mathrm{MeV}$. We match the values of $T$ from the two EoS in the temperature range from 150 to $170 \mathrm{MeV}$ and apply the lattice EoS exclusively for higher temperatures to avoid discontinuities in the evolution. Note that in the lattice $\operatorname{EoS} \mu_{B}$ is always zero.

Finally we calculate the thermal dilepton emission rate for each cell. The dilepton yield per fourvolume and four-momentum is directly related to the imaginary part of the retarded electromagnetic current-current correlator as [3]

$$
\frac{\mathrm{d} N_{l l}}{\mathrm{~d}^{4} x \mathrm{~d}^{4} q}=-\frac{\alpha_{\mathrm{em}}^{2} L(M)}{\pi^{3} M^{2}} f^{\mathrm{B}}(q \cdot U ; T) \operatorname{Im} \Pi_{\mathrm{em}}^{(\mathrm{ret})}\left(M, \vec{q} ; \mu_{B}, T\right) .
$$

In the hadronic domain, the correlator $\Pi_{\mathrm{em}}^{(\mathrm{ret})}$ is proportional to the vector-meson fields respectively their spectral functions under the hypothesis that vector-meson dominance is valid. For the present approach we calculate thermal emission for all cells with a temperature above $50 \mathrm{MeV}$. For lower temperatures, where the densities are so low that the assumption of a thermal emission becomes questionable and the tabulated EoS we use is less accurate, we directly take the $\rho$ mesons from the UrQMD transport calculation and let them decay to dileptons. As we only calculate either thermal or non-thermal contribution for each cell, we avoid double-counting.

It is important to note that equation (4) is only valid for the case that the system is in chemical equilibrium with regard to the pion density and no pion chemical potential builds up. However, it has been discussed whether this is really an appropriate description. Some authors have argued that an over-dense pionic system is created in the initial stage of the collision and that the fireball remains out of equilibrium due to the long relaxation time [31, 32]. In other models $\mu_{\pi}$ becomes finite after the number of pions is fixed at the chemical freeze-out but the system further cools and extends $[10,33]$. In any case, such a chemical off-equilibrium requires an additional fugacity factor $z_{\pi}^{n}=\exp \left(n \mu_{\pi} / T\right)$ in the foregoing equation, with $n=2$ in the case of the $\rho$ contribution and $n=3,4,5$ for multi-pion interactions (see section 3). This is due to the fact that equation (4) is independent of the hadronic initial and final states as only chemical potentials of conserved charges are considered for its derivation, for which $Q_{i}=Q_{f}$ is always complied. However, since the pion number $N_{\pi}$ is not a conserved quantity, this assumption is inappropriate in case of a finite pion chemical potential which implies that generally $N_{\pi, i}-N_{\pi, f} \neq 0$. Note that for the quark-gluon plasma $\mu_{\mathrm{B}}=\mu_{\pi}=0$ is assumed in the present work. We will investigate the influence of a pion chemical potential on the dilepton rates in these proceedings. For this, we extract $\mu_{\pi}$ for each cell in Boltzmann approximation according to

$$
\mu_{\pi}=T \cdot \ln \left(\frac{2 \pi^{2} n_{\pi}}{g_{\pi} T m^{2} \mathrm{~K}_{2}(m / T)}\right)
$$

where $\mathrm{K}_{2}$ denotes the Bessel function of the second kind, $n_{\pi}$ the pion density in the cell and $g_{\pi}$ the degeneracy factor (which is 3 for pions).

A more detailed description of the coarse-graining approach and how it is used to calculate thermal dilepton emission can be found in reference [34].

\section{In-medium Spectral Functions}

Once the thermodynamic properties of the cell are known, it is important to describe the dependency of the spectral functions on these parameters. Note that the focus is on the $\rho$ here, as the $\omega$ and $\phi$ mesons have a much longer lifetime and only show small medium modifications at SPS energies. As 
calculations from first principles in the non-perturbative hadronic domain of QCD are difficult and a measurement of the spectral shape is only possible for the vacuum (via $e^{+} e^{-}$-collisions), one has to rely on hadronic models for this purpose. There exist several approaches for spectral functions of vector mesons, but only few of those consider both the influence of finite temperature and finite density on the in-medium self-energy of the $\rho$ meson. In the present work we employed and compare the work by Rapp and collaborators [35] who used hadronic many-body theory to implement medium modifications (Rapp SF), and another more model-independent approach by Eletsky et al. [36] where empirical scattering amplitudes are used to determine the spectral properties (Eletsky SF).

The Rapp SF includes three different contributions for the calculation of the total self-energy: Polarizations of the pion cloud $\left(\Sigma_{\pi \pi}\right)$, interactions with mesons, i.e. pions, kaons and rhos $\left(\Sigma_{\rho M}\right)$ and the resonant scattering with nucleons respectively the most abundant baryonic resonances $\left(\Sigma_{\rho B}\right)$ [37]. The calculation also takes anti-baryons into account as the interaction with the $\rho$ is the same as for a baryon. For this purpose the effective baryon density $\rho_{\text {eff }}=\rho_{N}+\rho_{\bar{N}}+0.5\left(\rho_{B^{*}}+\rho_{\bar{B}^{*}}\right)$ instead of the baryochemical potential enters the Rapp SF as $\mu_{B}$ only accounts for the net baryon density. Accordingly, the in-medium propagator takes the form

$$
D_{\rho}(M, q ; T)=\left[M^{2}-m_{\rho}^{0}-\Sigma_{\pi \pi}-\Sigma_{\rho M}-\Sigma_{\rho B}\right]^{-1} .
$$

In the approach by Eletsky et al. the spectral behavior is calculated under the more simplified assumption of a non-interacting gas of pions and nucleons. Consequently, only the $\rho-\pi$ and $\rho-N$ scattering amplitudes enter the in-medium self-energy contribution. The idea here is that the medium modification of a particle's spectral function can be related to the forward scattering amplitude $f$ of this particle on the constituents of the medium [38] via the optical theorem. While the low-energy amplitudes are saturated with resonances from the partial-wave analysis by Manley and Saleski [39] plus a background contribution, a Regge fit is performed for high-energy scatterings. For the $\rho$ meson scattering from hadron $a$ in the heat bath follows a contribution to the in-medium self energy of the form

$$
\Sigma_{\rho a}(E, p)=-4 \pi \int \frac{\mathrm{d}^{3} k}{(2 \pi)^{3}} n_{a}(\omega) \frac{\sqrt{s}}{\omega} f_{\rho a}(s) .
$$

Note that equation (7) is a low-density expansion (similar to a virial expansion) and therefore fully applicable only if the system is dilute enough. However, in the hot and dense stage it is questionable whether this assumption still holds. Additionally the optical analogy - on which the above expression is based - requires the particle's wavelength to be significantly smaller than the average distance between the constituents of the medium and the scattering angle to be small. In contrast to the approach by Rapp et al, the influence of kaons, excited baryons and hyperons on the $\rho$ self energy is neglected here. Furthermore, no anti-baryons are included in the calculation. Besides the $\rho$, it will be important to include also the other relevant thermal sources (although we focus on the $\rho$ part here). At the collision energy considered here, the creation of a deconfined phase of free quarks and gluons is possible. In this case dilepton production is possible via the reaction $q \bar{q} \rightarrow l^{+} l^{-}$, i.e. a quark and an anti-quark can annihilate into a virtual photon respectively a lepton pair. To determine the QGP dilepton yield we use an extrapolation of rates obtained from lattice calculations [40] to non-zero three momentum [41]. In the hadronic phase, a significant contribution to the dilepton yield will also originate from multi-pion interactions. Following the approach of Dey, Eletsky and Ioffe [42] this contribution is determined taking the mixing of the vector and axial-vector currents into account.

\section{Results}

For the results presented here we used as input 1000 events of In+In collisions at a beam energy of $E_{\text {lab }}=158 \mathrm{AGeV}$ simulated with the most recent version 3.4 of the UrQMD model. We 

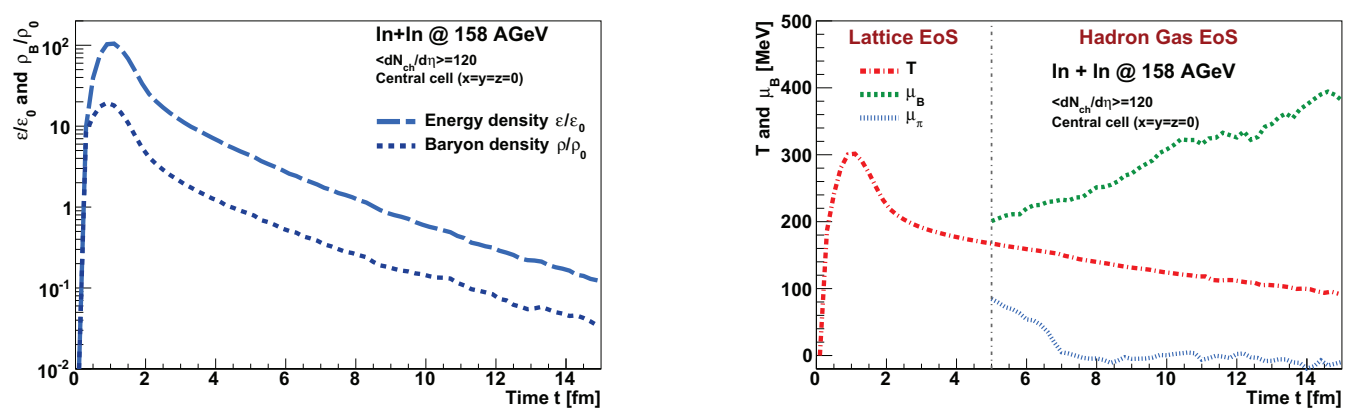

Figure 1. Time evolution of the energy and baryon densities, $\varepsilon$ and $\rho_{B}$, in units of the ground-state densities (left) and for temperature $T$ as well as the baryon and pion chemical potentials $\mu_{B}$ and $\mu_{\pi}$ (right) for In+In collisions at $158 \mathrm{AGeV}$. The results shown here are from the central cell of the coarse-graining grid, i.e. for a small volume around $x=y=z=0$.

chose an impact parameter distribution from 0 to $8.5 \mathrm{fm}$, corresponding to a charged particle number $\left\langle\mathrm{d} N_{\mathrm{ch}} / \mathrm{d} \eta\right\rangle=119$ which is very close to the experimentally measured value.

Figure 1 shows the time evolution for the central cell of the coarse-graining grid, i.e. for a small volume around $x=y=z=0$. While the left plot presents the energy and baryon densities in units of the ground state densities, the right plot depicts the evolution of temperature $T$ as well as the baryon and pion chemical potentials $\mu_{B}$ and $\mu_{\pi}$. We obtain a maximum energy density that is 100 times the ground state density and a baryon density reaching up to 20 times $\rho_{0}$. The maxima are reached roughly $1 \mathrm{fm} / \mathrm{c}$ after the begin of the collision. Similarly, as is visible from the right plot, the temperature reaches its highest value of roughly $300 \mathrm{MeV}$ after $1 \mathrm{fm} / \mathrm{c}$ and drops off again afterwards. However, even after $15 \mathrm{fm} / \mathrm{c}$ the cell has still a temperature of $100 \mathrm{MeV}$. However, one has to bear in mind that here in the center of the grid one obtains the locally highest densities which are not representative for the whole colliding system. Therefore this behaviour is mainly a specific property of the central cell, more peripherally the temperature is significantly lower. The dotted vertical line around $t=5 \mathrm{fm} / c$ denotes the transition from the lattice EoS, which is used for temperatures above $170 \mathrm{MeV}$, to the hadron gas. As we assume $\mu_{\mathrm{B}}=\mu_{\pi}=0$ in the QGP phase, we get values for the chemical potentials only after the temperature temperatures has fallen below $T_{c}$. While the baryon chemical potential then slowly increases from a value of $200 \mathrm{MeV}$ to $400 \mathrm{MeV}$ we observe an initially high pion chemical potential which drops to values around zero in the course of the evolution. The latter effect is due to the non-equilibrium nature of the underlying transport dynamics where many pions are created at the beginning of the collision, especially via string excitation. However, note once again that the central cell is not necessarily representative for the evolution of the whole system.

The dilepton invariant mass spectra are presented in Figure 2. The left plot shows the result as obtained with the coarse-graining approach and the Rapp spectral function for the $\rho$. The total yield includes the thermal $\rho$ contribution as well as the yield from multi-pion interactions, from the quarkgluon plasma and a transport $\rho$ contribution from cold cells. The model results are contrasted to the experimental data from the NA60 collaboration [43]. The description of the measured yield is quite good within the error bars, especially the low-mass excess can be explained with a broadening of the $\rho$ spectral function as compared to the vacuum shape. The non-thermal $\rho$ is here rather negligible in relation to the thermal contribution. Also the higher mass region above $1 \mathrm{GeV} / c^{2}$, which is dominated by the contributions from multi-pion interactions and the QGP, can be described with the model. 

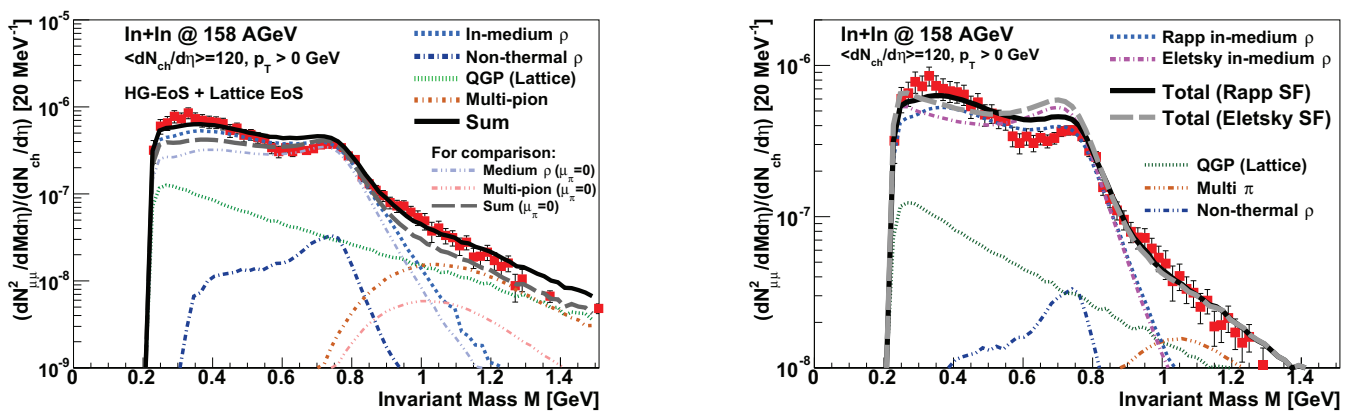

Figure 2. (Left) Invariant-mass spectrum of the dilepton excess yield for In+In collisions at $158 \mathrm{AGeV}$ as obtained within the coarse-graining approach. We show the thermal $\rho$ contribution (Rapp SF) as well as the yield from multi-pion interactions and from the quark-gluon plasma. The model results are compared to the experimental data from the NA60 collaboration [43]. For comparison, we also show the dilepton yield under the assumption of a vanishing pion chemical potential. (Right) Same as in the left plot, but here resulting yields from the two $\rho$ spectral functions by Rapp and Eletsky are compared to each other.

For comparison, we also show the dilepton yield for the assumption of a vanishing pion chemical potential. This results in an underestimation of the dilepton yield in the region from 0.2 to $0.4 \mathrm{GeV} / \mathrm{c}^{2}$ by a factor of two. Note that this difference is primarily caused by the fugacity factor in equation (4) which might easily double the emission rate in the presence of a large pion chemical potential, whereas the explicit dependence of the Rapp spectral function on $\mu_{\pi}$ is rather moderate. The large effect on the low-mass tail is due to the fact that the pion chemical potential reaches its highest values early in the evolution, when the baryon density is still very high, which is known to be the main cause of the low-mass enhancement $[6,7,34]$. A significantly lower yield from the multi-pion contribution is obtained as well for $\mu_{\pi}=0$, so that in the mass range around $1 \mathrm{GeV} / c^{2}$ the theoretical prediction is clearly below the data then.

The comparison between the results with the two different approaches for the $\rho$ spectral functions are shown in the right plot of Figure 2. While the peak at the pole mass of the $\rho$ meson is more pronounced for the Eletsky SF, a stronger dilepton yield shows up in the lower mass range from 0.3 to $0.5 \mathrm{GeV} / \mathrm{c}^{2}$ when using the Rapp SF. In general it accounts for a stronger melting of the peak due to baryonic effects for the spectral function from hadronic many-body theory. Naturally one will see a stronger density effect than within the low-density expansion of the empirical scattering amplitudes, as used in the approach of Eletsky. Besides, the Eletsky SF does not include all the effects considered within the Rapp SF as only $\rho-N$ and $\rho-\pi$ scatterings are considered. It has also been pointed out that the Eletsky SF (where the amplitudes are evaluated on-shell only) misses the off-shell contributions from sub-threshold baryon resonances, that contribute substantially to the low-mass enhancement of the $\rho[6]$.

\section{Conclusions \& Outlook}

We have presented calculations of the thermal dilepton yield for In+In collisions at top SPS energy from coarse-grained microscopic dynamics. The results show a good agreement with the measurements of the NA60 collaboration. While both in-medium spectral functions for the $\rho$ meson have proven to be in qualitative accordance with the data, the Rapp SF shows a stronger broadening of the 
spectral shape and gives a significantly better quantitative description. The present study also shows that within the coarse-graining approach the assumption of a finite pion chemical potential is crucial to describe the data. This is in agreement with other investigations that included a pion chemical potential $[9,44]$. When comparing our results to the outcome of similar investigations, one finds that quite different theoretical approaches to the reaction dynamics finally result in the similar dilepton spectra. For example the fireball approach has a significantly different evolution of $T, \mu_{\mathrm{B}}$ and $\mu_{\pi}$ but the resulting dilepton excess spectrum shows no large deviations from our result (compare [9]). Furthermore the relative strengths of the various contributions vary from the coarse-graining results. It therefore becomes clear that the time-integral character of the dilepton spectrum is not only a challenge for theory but also makes it difficult to discriminate between the models. Nevertheless, there is general agreement in line with our findings that hadronic modifications of the $\rho$ spectral function are the main source of the low-mass dilepton excess as observed at SPS energies. The comparison of the two spectral functions indicates that for a full description of the medium modifications several hadronic interactions have to be taken into account, as in the hadronic many-body calculation.

\section{Acknowledgments}

The authors acknowledge R. Rapp for providing the spectral function and for many fruitful discussions. This work was supported by BMBF, HICforFAIR and the H-QM.

\section{References}

[1] E.V. Shuryak, Phys. Lett. B 78, 150 (1978)

[2] G. Domokos, J.I. Goldman, Phys. Rev. D 23, 203 (1981)

[3] L.D. McLerran, T. Toimela, Phys. Rev. D 31, 545 (1985)

[4] T. Hatsuda, S.H. Lee, Phys. Rev. C 46, 34 (1992)

[5] F. Klingl, N. Kaiser, W. Weise, Nucl. Phys. A 624, 527 (1997)

[6] R. Rapp, J. Wambach, Adv. Nucl. Phys. 25, 1 (2000)

[7] S. Leupold, V. Metag, U. Mosel, Int. J. Mod. Phys. E 19, 147 (2010)

[8] C. Gale, J.I. Kapusta, Phys. Rev. C 35, 2107 (1987)

[9] H. van Hees, R. Rapp, Phys. Rev. Lett. 97, 102301 (2006)

[10] H. van Hees, R. Rapp, Nucl. Phys. A 806, 339 (2008)

[11] G. Vujanovic, C. Young, B. Schenke, R. Rapp, S. Jeon et al., Phys. Rev. C 89, 034904 (2014)

[12] R. Ryblewski, M. Strickland (2015), arXiv: 1501.03418 [nucl-th]

[13] E. Santini, J. Steinheimer, M. Bleicher, S. Schramm, Phys. Rev. C 84, 014901 (2011)

[14] M. Thomere, C. Hartnack, G. Wolf, J. Aichelin, Phys. Rev. C 75, 064902 (2007)

[15] E. Bratkovskaya, W. Cassing, O. Linnyk, Phys. Lett. B 670, 428 (2009)

[16] O. Linnyk, E. Bratkovskaya, V. Ozvenchuk, W. Cassing, C. Ko, Phys. Rev. C 84, 054917 (2011)

[17] E. Bratkovskaya, J. Aichelin, M. Thomere, S. Vogel, M. Bleicher, Phys. Rev. C 87, 064907 (2013)

[18] J. Weil, H. van Hees, U. Mosel, Eur. Phys. J. A 48, 111 (2012)

[19] S. Vogel, H. Petersen, K. Schmidt, E. Santini, C. Sturm et al., Phys. Rev. C 78, 044909 (2008)

[20] K. Schmidt, E. Santini, S. Vogel, C. Sturm, M. Bleicher et al., Phys. Rev. C 79, 064908 (2009)

[21] W. Cassing, E. Bratkovskaya, R. Rapp, J. Wambach, Phys. Rev. C 57, 916 (1998)

[22] E. Bratkovskaya, W. Cassing, R. Rapp, J. Wambach, Nucl. Phys. A 634, 168 (1998) 
[23] B. Schenke, C. Greiner, Phys. Rev. C 73, 034909 (2006)

[24] P. Huovinen, M. Belkacem, P.J. Ellis, J.I. Kapusta, Phys. Rev. C 66, 014903 (2002)

[25] S.A. Bass, M. Belkacem, M. Bleicher et al., Prog. Part. Nucl. Phys. 41, 255 (1998)

[26] M. Bleicher, E. Zabrodin, C. Spieles, S.A. Bass, C. Ernst et al., J. Phys. G 25, 1859 (1999)

[27] H. Petersen, M. Bleicher, S.A. Bass, H. Stöcker (2008), arXiv: 0805.0567 [hep-ph]

[28] W. Florkowski, M. Martinez, R. Ryblewski, M. Strickland, Nucl. Phys. A 904-905, 803c (2013)

[29] D. Zschiesche, S. Schramm, J. Schaffner-Bielich et al., Phys. Lett. B 547, 7 (2002)

[30] M. He, R.J. Fries, R. Rapp, Phys. Rev. C 85, 044911 (2012)

[31] M. Kataja, P. Ruuskanen, Phys. Lett. B 243, 181 (1990)

[32] H. Bebie, P. Gerber, J. Goity, H. Leutwyler, Nucl. Phys. B 378, 95 (1992)

[33] P.F. Kolb, R. Rapp, Phys. Rev. C 67, 044903 (2003)

[34] S. Endres, H. van Hees, J. Weil, M. Bleicher (2014), arXiv: 1412 .1965 [nucl-th]

[35] R. Rapp, J. Wambach, Eur. Phys. J. A 6, 415 (1999)

[36] V.L. Eletsky, M. Belkacem, P. Ellis, J.I. Kapusta, Phys. Rev. C 64, 035202 (2001)

[37] R. Rapp, Phys. Rev. C 63, 054907 (2001)

[38] V. Eletsky, B. Ioffe, J.I. Kapusta, Eur. Phys. J. A 3, 381 (1998)

[39] D. Manley, E. Saleski, Phys. Rev. D 45, 4002 (1992)

[40] H.T. Ding, A. Francis, O. Kaczmarek, F. Karsch et al., Phys. Rev. D 83, 034504 (2011)

[41] R. Rapp, Adv. High Energy Phys. 2013, 148253 (2013)

[42] M. Dey, V. Eletsky, B.L. Ioffe, Phys. Lett. B 252, 620 (1990)

[43] R. Arnaldi et al. (NA60 Collaboration), Phys. Rev. Lett. 96, 162302 (2006)

[44] P. Koch, Phys. Lett. B 288, 187 (1992) 
